\title{
Spectra of extreme GLEs derived using neutron monitor network records
}

\author{
Alexander Mishev* \\ Space Climate Research Unit, University of Oulu, Finland; Sodankylä Geophysical Observatory, \\ University of Oulu, Finland. \\ E-mail: alexander.mishev@oulu.fi \\ Ilya Usoskin \\ Space Climate Research Unit, University of Oulu, Finland; Sodankylä Geophysical Observatory, \\ University of Oulu, Finland. \\ E-mail: ilya.usoskin@oulu.fi
}

Study of solar energetic particles (SEPs) provides important knowledge to understand their acceleration and propagation in the interplanetary space. In most cases the maximum energy of SEPs is several tens of $\mathrm{MeV} /$ nucleon, but in some cases it exceeds $100 \mathrm{MeV} /$ nucleon or even reaches several GeV/nucleon. In that case the energy is sufficient to generate an extensive air shower in the Earth's atmosphere, whose secondary particles reach the ground, subsequently registered by ground based detectors, specifically neutron monitors (NMs). This particular class of events is known as ground level enhancements (GLEs). Historically the strongest GLE \#5 was registered on 23 February 1956, with peak increase of the count rate of NMs above $5000 \%$. The solar cycle 23 provided several strong GLEs, including the second largest event in the observational history observed on 20 January 2005 (GLE \#69). Here, we derive the spectral and angular features of the strongest GLEs using data from the world-wide NM network. We model the global NM network response including the particle propagation in the Earth's magnetosphere and atmosphere. The method includes several consecutive steps: detailed computation of asymptotic cones and rigidity cut-off of each NM station used in the analysis, an initial guess of the inverse problem by assuming the apparent source position location in a convenient way, application of the NM yield function for detector response modelling and optimization procedure in order to derive spectral and angular characteristics of SEPs. The SEP spectra and pitch angle distributions are obtained in their dynamical development throughout the events.

36th International Cosmic Ray Conference - ICRC 2019-

24 July-1 August, 2019

Madison, United States

\footnotetext{
${ }^{*}$ Speaker.
} 


\section{Introduction}

Sporadically, violent energy releases occur on the Sun, that are related to eruptive process as solar flares and coronal mass ejections (CMEs). Such events lead to transient phenomena in the interplanetary space and in particular produce solar energetic particles (SEPs) [1, 2]. Occasionally, the energy of SEPs can reach about $1 \mathrm{GeV} /$ nucleon and even higher [3, 4], leading to development of an extensive air shower of secondary particles in the Earth's atmosphere. The secondaries in the cascade can increase the count rate of ground-based detectors, specifically neutron monitors (NMs). This particular class of events is called ground-level enhancements (GLEs) [5,6]. They can be considered as extreme class of SEP events [7] and their study is important in order to clarify the particle acceleration on the Sun [3, 8] as well as space weather applications [9].

GLEs are routinely studied using NM records $[10,11]$, the data archived at the International GLE database https://gle.oulu.fi [12]. There are 72 registered events so far, quantified as NM count rate increase above the background due to galactic cosmic rays (GCRs). The two strongest events are GLE \#5 on 23 February 1956 and GLE \#69 on 20 January 2005. These events are remarkable with their considerably greater NM count rate increase compared to the bulk of events. In this study we derive, on the basis of NM records, the rigidity spectra and anisotropy distribution of GLE \#5 (Fig.1a) and GLE \#69 (Fig.1b).

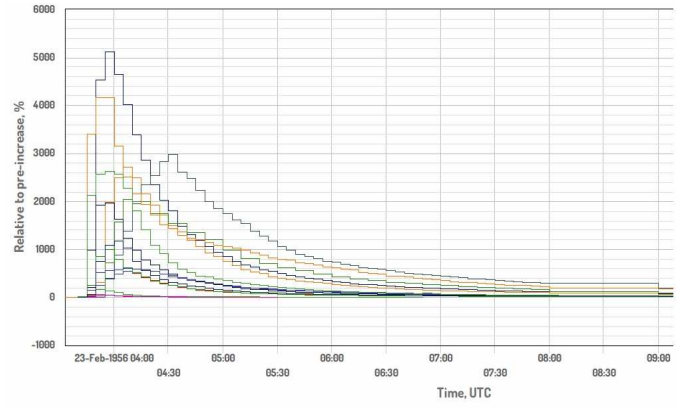

(a) GLE \#5 on 23 February 1956.

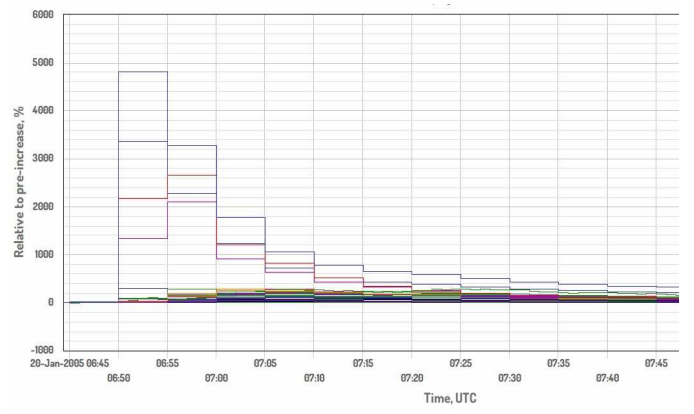

(b) GLE \#69 on 20 January 2005.

Figure 1: NM count rate variation during two extreme GLEs. The horizontal axis represents UT time, while the vetical axis represents NM count rate increase in \%.

\section{Model for analysis of GLEs using global NM records}

A realistic modelling of the global NM response allows one to assess the GLE particles spectra and anisotropy [10]. Here, we use a model described with great details in $[13,14,15,16]$. In general, the method involves computation of cut-off rigidities and asymptotic viewing directions of NMs, making an initial guess of the unfolding procedure of model over the experimental records $[17,18]$ and optimization of a set of unknown parameters over the difference of experimental and modelled NM responses. The count rate of a NM is modelled using a NM yield function:

$$
N\left(P_{\text {cut }}\right)=\int J(P, t) S(P) G(\alpha(P, t)) A(P) d P
$$


where $J(P, t)$ is the rigidity spectrum of the primary CR of galactic or solar origin at given moment $t, S(P)$ is the specific NM yield function, $G(\alpha(P, t))$ is the anisotropy i.e. the pitch angle distribution (PAD), $\mathrm{A}(\mathrm{P})$ is a discrete function, which accounts for allowed $(A(P)=1)$, accordingly forbidden trajectories $(A(P)=0)$. Here, we used a recently computed specific yield function [19], which provides a very good agreement with experimental data and models [20, 21, 22, 23] and it is suitable for GLE analysis [22, 23]. The background due to GCRs was computed using the force-field model $[24,25,26]$, where the modulation potential is computed similarly to [27]. The asymptotic trajectories and cut-off rigidities of NMs were computed using MAGNETOCOSMICS code [28] employing a combination of IGRF model as internal field and the Tsyganenko 89 model [29] as the external field, respectively.

The spectra of GLE particles are modelled with a modified power-law rigidity spectrum [30, 31]:

$$
J(P)=J_{0} P^{-(\gamma+\delta \gamma(P-1))}
$$

where $J(P)$ is the particle flux with a given rigidity $P$ in $[\mathrm{GV}], \gamma$ is the power-law spectral exponent at rigidity $\mathrm{P}=1 \mathrm{GV}$, accordingly $\delta \gamma$ in $\left[\mathrm{GV}^{-1}\right]$ is the rate of the spectrum steepening. Note that in the case of significant steepening $\delta \gamma$, the modified power-law spectrum (2.2) is very similar to exponential shape.

The PAD is modelled with a Gauss distribution:

$$
G(\alpha(P)) \sim \exp \left(-\alpha^{2} / \sigma^{2}\right)
$$

where $\alpha$ is the pitch angle and $\sigma$ corresponds to the width of the distribution.

\section{Spectra and PAD of SEPs during GLE \#5 and GLE \#69}

The GLE \#5 was the largest event observed so far. It occurred on 23 February 1956 and was registered by ground-based detectors (NMs, ionization chambers and muon telescopes). This event was associated with strong solar flare occurred at 03:31 UT in the active region with coordinated $25^{\circ} \mathrm{N}$ and $85^{\circ} \mathrm{W}$, near to the west limb. The event was observed during the recovery phase of a large Forbush decrease, which was explicitly considered, specifically for low latitude stations and late phase of the event. The GLE \#5 was very anisotropic, since a large asymmetry between Leeds (LEED), Stockholm (STHM) and Weissenau (WEIS) NMs on one hand, and Chicago (CHGO) and Ottawa (OTWA) NMs on the other hand was observed. Thus, the stations in Europe revealed rapid and very large NM count rate increases, while those in North America were with considerably delayed maximum and smaller count rate enhancements (Fig.1a). This observation can be explained assuming a narrow SEP flux and considering the different asymptotic directions of the NMs (Fig.2).

Using NM data and the model described in Section 2, we derived the GLE particles rigidity spectra and PAD throughout the event, as depicted in Fig.3. The derived spectra (Fig.3a) are very hard, specifically during the event onset and initial phase, accordingly the derived PAD was very narrow (Fig.3b). The spectra remained hard during the whole event, specifically during the initial and main phase of the event (when a narrow distribution, SEP flux rise and exponentially like spectra were derived). The SEP spectra was constantly softening throughout the event, specifically 
during the late phase, but an essential steepening remained even in the late phase. Accordingly, the PAD was very narrow during the event onset and constantly widened out.

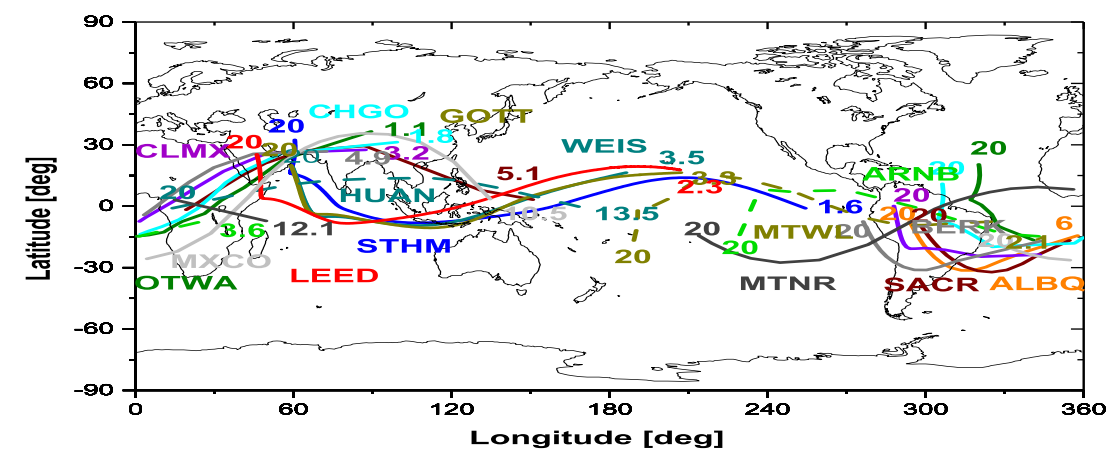

Figure 2: Asymptotic directions of NMs during GLE \#5.
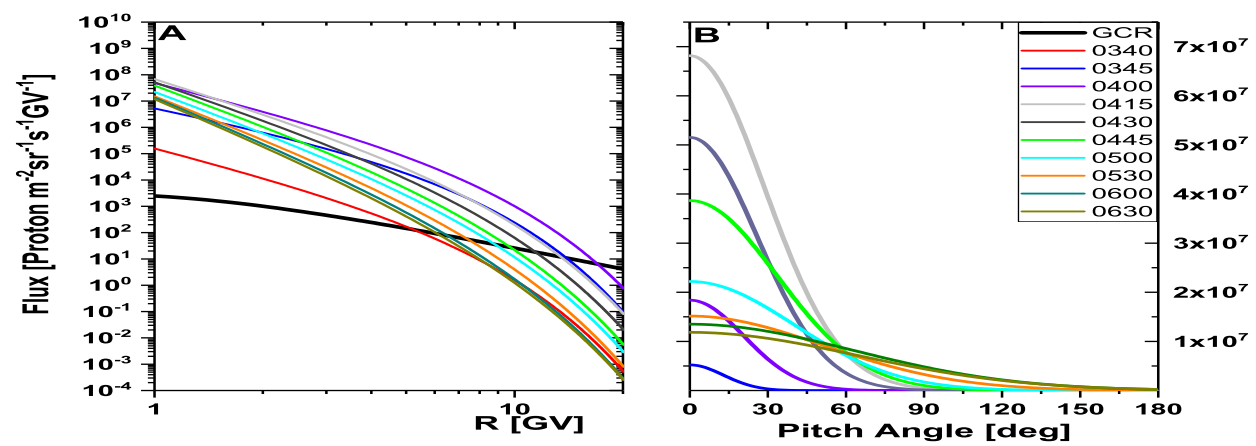

Figure 3: Rigidity spectra (panel a) and PAD (panel b) during GLE \#5.

The GLE \#69 event on 20 January 2005 occurred in complex magnetospheric conditions and also during the recovery phase of a Forbush decrease. A large anisotropy was observed, since South Pole (SOPO), Terre Adelie (TERA) and McMurdo (MCMD) stations registered considerably greater NM count rate increases than the other NMs.

According to our analysis, we observed SEPs arriving within two fluxes. A very narrow flux 1 with a very hard spectrum, was responsible for the giant NM count rate increases at SOPO, TERA and MCMD NMs. Flux 1 was characterized by a hard spectrum (Fig.5a) with considerable steepening, a narrow PAD (Fig.5b), a very high peak SEP intensity, specifically during event onset and initial phase, which rapidly dropped. Flux 1 vanished after 08:00 UT.

The second GLE particles flux arrived at about $60^{\circ}$ apart from maximum PAD of flux 1 . It was characterized by a softer, but still a hard spectrum (Fig.6a), with a smaller steepening, a wider PAD (Fig.6b), a very high peak SEP intensity during the event initial phase, which constantly decreased. During the main and late phase of the event, the steepness vanished and after 10:30 UT the SEP spectra were pure power-law. The derived PAD was relatively narrow during the event onset and initial phase, but rapidly broaden out (Fig.6). Moreover, the SEP flux was nearly isotropic during the late phase of the event (Fig.7). 


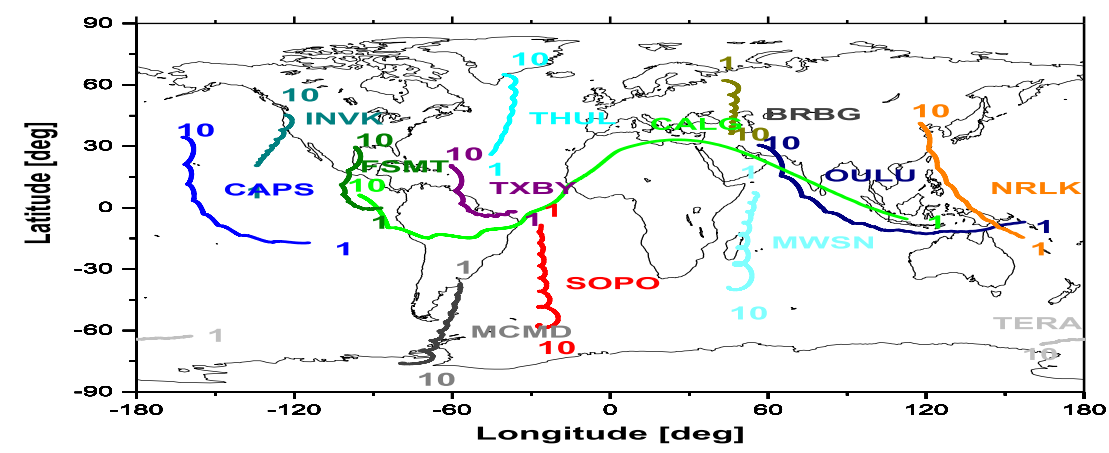

Figure 4: Asymptotic directions of selected NMs during GLE \#69.

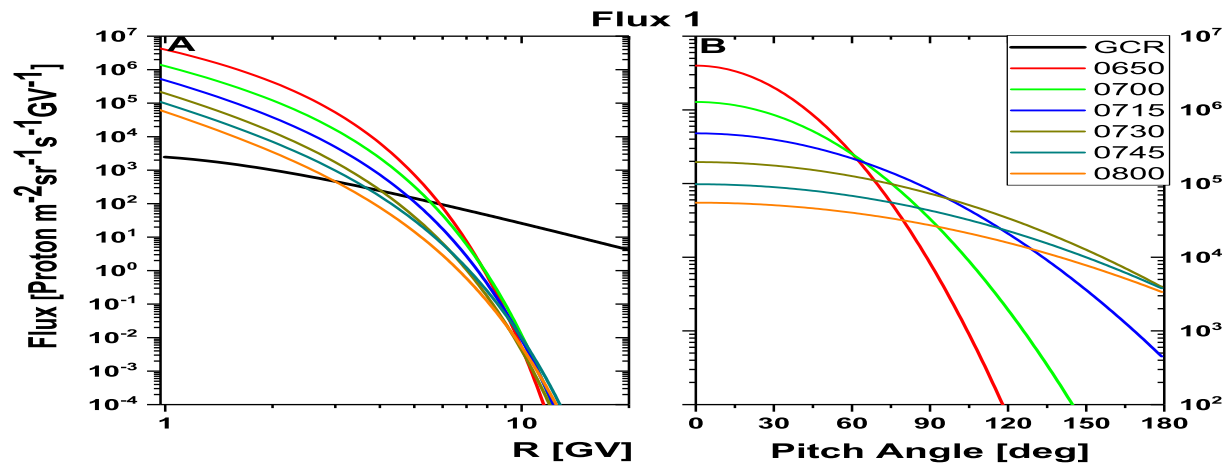

Figure 5: Spectra (panel a) and PAD (panel b) of Flux 1 of GLE particles during GLE \#69.

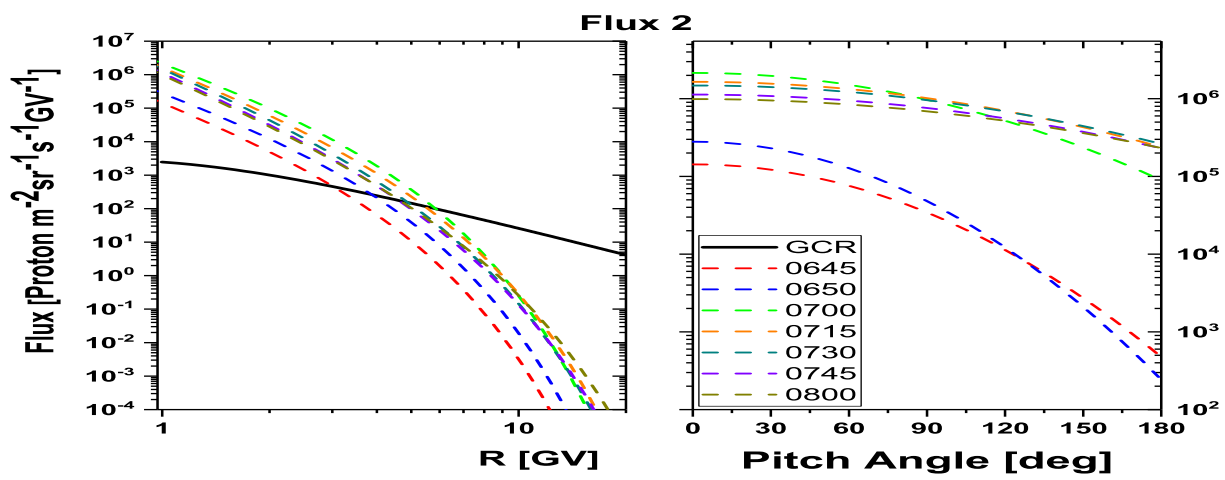

Figure 6: Spectra (panel a) and PAD (panel b) of Flux 2 of GLE particles during initial phase of GLE \#69.

\section{Conclusion}

Using NM records, we derived the rigidity spectra and PAD of SEPs during the two strongest GLEs. The SEP characteristics are derived by a detailed modelling of ground-based NM data. In the case of GLE \#5 the best fit of the global NM network response reveals a very hard spectrum and single Gaussian PAD. In the initial phase of the event the derived spectrum was with almost 

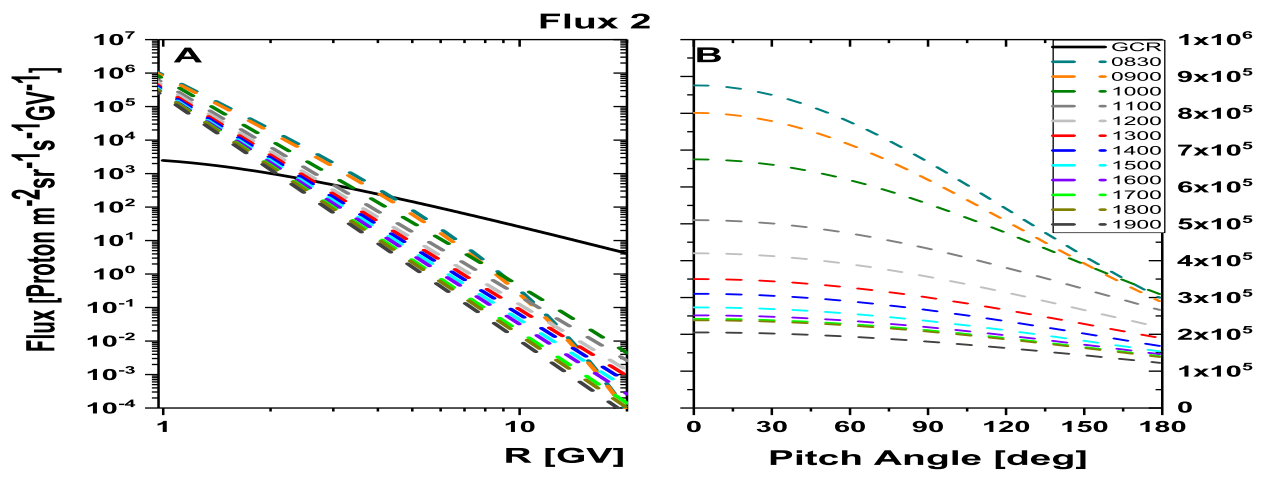

Figure 7: Spectra (panel a) and PAD (panel b) of Flux 2 of GLE particles during main and late phase of GLE \#69.

exponential shape. A strong anisotropy, specifically during the initial phase of the event was derived, which gradually decreased in the course of the event, but remained important throughout the whole event.

In the case of GLE \#69 we derived SEP characteristics, which depict a very complicated scenario, namely a two independent particle fluxes arriving almost simultaneously. Flux 1, characterized by a beam like PAD and very hard spectrum, vanished after the initial phase of the event, while flux 2, characterized by a wider PAD and a softer spectrum remained throughout the whole event. The derived results are with fair agreement with some previous studies, specifically $[32,33]$.

The derived spectra and PAD of the two strongest GLEs make it possible to probe different scenarios of relativistic SEPs acceleration and are very important to study radiation effects similarly to $[34,35]$.

\section{Acknowledgements}

This work was supported by the Academy of Finland (project 307411, Center of Excellence ReSoLVE) We acknowledge the International Space Science Institute support to International Team 441: High EneRgy sOlar partICle Events Analysis (HEROIC). We acknowledge neutron monitor database (NMDB) and all the colleagues and PIs from the neutron monitor stations, who kindly provided the data used in this analysis.

\section{References}

[1] R. Vainio, L. Desorgher, D. Heynderickx, M. Storini, E. Flückiger, R. Horne, G. Kovaltsov, K. Kudela, M. Laurenza, S. McKenna-Lawlor, H. Rothkaehl and I. Usoskin, Dynamics of the Earth's particle radiation environment, Space Science Reviews 147 (2009), no. 3-4 187-231.

[2] M. Desai and J. Giacalone, Large gradual solar energetic particle events, Living Reviews in Solar Physics 13 (2016), no. 13.

[3] M. Aschwanden, GeV particle acceleration in solar flares and ground level enhancement (GLE) events, Space Science Reviews 171 (2012), no. 1-4 3-21. 
[4] D. Reames, The two sources of solar energetic particles, Space Science Reviews 175 (2013), no. 1-4 53-92.

[5] M. A. Shea, I. Zaljubovsky, M. Wada and A. Inoue, A suggested standardized format for cosmic ray ground-level event data, in Proc. of 19th ICRC La Jolla, USA, 11 - 23 August 1985, vol. 5, pp. 510-513, 1985.

[6] S. Poluianov, I. Usoskin, A. Mishev, A. Shea and D. Smart, GLE and sub-GLE redefinition in the light of high-altitude polar neutron monitors, Solar Physics 292 (2017), no. 11176.

[7] N. Gopalswamy, S. Yashiro, N. Thakur, P. Mäkelä, H. Xie and S. Akiyama, The 2012 July 23 backside eruption: An extreme energetic particle event?, Astrophysical Journal 833 (2016), no. 2216.

[8] K.-L. Klein and S. Dalla, Acceleration and propagation of solar energetic particles, Space Science Reviews 212 (2017), no. 3-4 1107-1136.

[9] A. Mishev and P. Jiggens, Preface to measurement, specification and forecasting of the solar energetic particle (SEP) environment and ground level enhancements (GLEs), J. Space Weather Space Clim. 9 (2019) E1.

[10] M. Shea and D. Smart, Possible evidence for a rigidity-dependent release of relativistic protons from the solar corona, Space Science Reviews 32 (1982) 251-271.

[11] L. Dorman, Cosmic Rays in the Earth's Atmosphere and Underground. Kluwer Academic Publishers, Dordrecht, 2004.

[12] I. Usoskin, A. Ibragimov, M. Shea and D. Smart, Database of ground level enhancements (GLE) of high energy solar proton events, Proceedings of Science, Proc. of 34th ICRC Hague, Netherlands, 30 July - 6 August 2015 (2015) 054.

[13] A. Mishev, L. Kocharov and I. Usoskin, Analysis of the ground level enhancement on 17 May 2012 using data from the global neutron monitor network, Journal of Geophysical Research 119 (2014) 670-679.

[14] A. Mishev and I. Usoskin, Analysis of the ground level enhancements on 14 July 2000 and on 13 December 2006 using neutron monitor data, Solar Physics 291 (2016), no. 4 1225-1239.

[15] A. Mishev, S. Poluianov and I. Usoskin, Assessment of spectral and angular characteristics of sub-GLE events using the global neutron monitor network, Journal of Space Weather and Space Climate 7 (2017) A28.

[16] A. Mishev, I. Usoskin, O. Raukunen, M. Paassilta, E. Valtonen, L. Kocharov and R. Vainio, First analysis of GLE 72 event on 10 September 2017: Spectral and anisotropy characteristics, Solar Physics 293 (2018) 136.

[17] A. Mishev and I. Usoskin, Erratum to:analysis of the ground level enhancements on 14 July 2000 and on 13 December 2006 using neutron monitor data, Solar Physics 291 (2016), no. 4 1579-1580.

[18] L. Kocharov, S. Pohjolainen, A. Mishev, M. Reiner, J. Lee, T. Laitinen, L. Didkovsky, V. Pizzo, R. Kim, A. Klassen, M. Karlicky, K.-S. Cho, D. Gary, I. Usoskin, E. Valtonen and R. Vainio, Investigating the origins of two extreme solar particle events: Proton source profile and associated electromagnetic emissions, Astrophysical Journal 839 (2017), no. 279.

[19] A. Mishev, I. Usoskin and G. Kovaltsov, Neutron monitor yield function: New improved computations, Journal of Geophysical Research 118 (2013) 2783-2788.

[20] A. Gil, I. Usoskin, G. Kovaltsov, A. Mishev, C. Corti and V. Bindi, Can we properly model the neutron monitor count rate?, J. Geophys. Res. 120 (2015) 7172-7178. 
[21] P.-S. Mangeard, D. Ruffolo, A. Sáiz, W. Nuntiyakul, J. Bieber, J. Clem, P. Evenson, R. Pyle, M. Duldig and J. Humble, Dependence of the neutron monitor count rate and time delay distribution on the rigidity spectrum of primary cosmic rays, Journal of Geophysical Research: Space Physics 121 (2016), no. 12 11,620-11,636.

[22] W. Nuntiyakul, A. Sáiz, D. Ruffolo, P.-S. Mangeard, P. Evenson, J. Bieber, J. Clem, R. Pyle, M. Duldig and J. Humble, Bare neutron counter and neutron monitor response to cosmic rays during a 1995 latitude survey, Journal of Geophysical Research: Space Physics 123 (2018), no. 9 7181-7195.

[23] S. A. Koldobskiy, V. Bindi, C. Corti, G. A. Kovaltsov and I. G. Usoskin, Validation of the Neutron Monitor Yield Function Using Data from AMS-02 Experiment 2011-2017, J. Geophys. Res. (Space Phys.) 124 (2019).

[24] L. Gleeson and W. Axford, Solar modulation of galactic cosmic rays, Astrophysical Journal 154 (1968) 1011-1026.

[25] R. Caballero-Lopez and H. Moraal, Limitations of the force field equation to describe cosmic ray modulation, Journal of Geophysical Research 109 (2004) A01101.

[26] I. Usoskin, K. Alanko-Huotari, G. Kovaltsov and K. Mursula, Heliospheric modulation of cosmic rays: Monthly reconstruction for 1951-2004, Journal of Geophysical Research 110 (2005), no. A12108.

[27] I. Usoskin, G. Bazilevskaya and G. Kovaltsov, Solar modulation parameter for cosmic rays since 1936 reconstructed from ground-based neutron monitors and ionization chambers, Journal of Geophysical Research 116 (2011) A02104.

[28] L. Desorgher, E. Flückiger, M. Gurtner, M. Moser, and R. Bütikofer, A GEANT 4 code for computing the interaction of cosmic rays with the earth's atmosphere, International Journal of Modern Physics A 20 (2005), no. A11 6802-6804.

[29] N. Tsyganenko, A magnetospheric magnetic field model with a warped tail current sheet, Planetary and Space Science 37 (1989), no. 1 5-20.

[30] J. Cramp, M. Duldig, E. Flückiger, J. Humble, M. Shea and D. Smart, The October 22, 1989, solar cosmic enhancement: ray an analysis the anisotropy spectral characteristics, Journal of Geophysical Research 102 (1997), no. A11 24 237-24 248.

[31] E. Vashenyuk, Y. Balabin, J. Perez-Peraza, A. Gallegos-Cruz and L. Miroshnichenko, Some features of the sources of relativistic particles at the sun in the solar cycles 21-23, Advances Space Research 38 (2006), no. 3 411-417.

[32] E. Vashenyuk, Y. Balabin, B. Gvozdevskii and S. Karpov, Relativistic solar protons in the event of January 20, 2005: Model studies, Geomagnetism and Aeronomy 46 (2006), no. 4 424-429.

[33] E. Vashenyuk, Y. Balabin and L. Miroshnichenko, Relativistic solar protons in the ground level event of 23 February 1956: New study, Advances in Space Research 41 (2008), no. 6 926-935.

[34] A. Mishev and I. Usoskin, Assessment of the radiation environment at commercial jet-flight altitudes during GLE 72 on 10 September 2017 using neutron monitor data, Space Weather 16 (2018), no. 12 1921-1929.

[35] A. Mishev and P. Velinov, Ion production and ionization effect in the atmosphere during the Bastille day GLE 59 due to high energy SEPs, Advances in Space Research 61 (2018), no. 1 316-325. 